\title{
The Geometric Interior in Real Linear Spaces
}

\author{
Karol Pąk \\ Institute of Informatics \\ University of Białystok \\ Poland
}

\begin{abstract}
Summary. We introduce the notions of the geometric interior and the centre of mass for subsets of real linear spaces. We prove a number of theorems concerning these notions which are used in the theory of abstract simplicial complexes.
\end{abstract}

MML identifier: RLAFFIN2, version: $\underline{7.11 .07 \quad 4.156 .1112}$

The papers [1], [6], [11], [2], [5], [3], [4], [13], [7], [16], [10], [14], [12], [8], [9], and [15] provide the terminology and notation for this paper.

\section{Preliminaries}

For simplicity, we adopt the following convention: $x$ denotes a set, $r, s$ denote real numbers, $n$ denotes a natural number, $V$ denotes a real linear space, $v, u$, $w, p$ denote vectors of $V, A, B$ denote subsets of $V, A_{1}$ denotes a finite subset of $V, I$ denotes an affinely independent subset of $V, I_{1}$ denotes a finite affinely independent subset of $V, F$ denotes a family of subsets of $V$, and $L_{1}, L_{2}$ denote linear combinations of $V$.

Next we state four propositions:

(1) Let $L$ be a linear combination of $A$. Suppose $L$ is convex and $v \neq \sum L$ and $L(v) \neq 0$. Then there exists $p$ such that $p \in \operatorname{conv} A \backslash\{v\}$ and $\sum L=$ $L(v) \cdot v+(1-L(v)) \cdot p$ and $\frac{1}{L(v)} \cdot \sum L+\left(1-\frac{1}{L(v)}\right) \cdot p=v$.

(2) Let $p_{1}, p_{2}, w_{1}, w_{2}$ be elements of $V$. Suppose that $v, u \in \operatorname{conv} I$ and $u \notin \operatorname{conv} I \backslash\left\{p_{1}\right\}$ and $u \notin \operatorname{conv} I \backslash\left\{p_{2}\right\}$ and $w_{1} \in \operatorname{conv} I \backslash\left\{p_{1}\right\}$ and 
$w_{2} \in \operatorname{conv} I \backslash\left\{p_{2}\right\}$ and $r \cdot u+(1-r) \cdot w_{1}=v$ and $s \cdot u+(1-s) \cdot w_{2}=v$ and $r<1$ and $s<1$. Then $w_{1}=w_{2}$ and $r=s$.

(3) Let $L$ be a linear combination of $A_{1}$. Suppose $A_{1} \subseteq \operatorname{conv} I_{1}$ and $\operatorname{sum} L=$ 1. Then

(i) $\quad \sum L \in$ Affin $I_{1}$, and

(ii) for every element $x$ of $V$ there exists a finite sequence $F$ of elements of $\mathbb{R}$ and there exists a finite sequence $G$ of elements of $V$ such that $\left(\sum L \rightarrow I_{1}\right)(x)=\sum F$ and len $G=\operatorname{len} F$ and $G$ is one-to-one and $\operatorname{rng} G=$ the support of $L$ and for every $n$ such that $n \in \operatorname{dom} F$ holds $F(n)=$ $L(G(n)) \cdot\left(G(n) \rightarrow I_{1}\right)(x)$.

(4) For every subset $A_{2}$ of $V$ such that $A_{2}$ is affine and conv $A \cap \operatorname{conv} B \subseteq A_{2}$ and conv $A \backslash\{v\} \subseteq A_{2}$ and $v \notin A_{2}$ holds conv $A \backslash\{v\} \cap \operatorname{conv} B=\operatorname{conv} A \cap$ conv $B$.

\section{The Geometric Interior}

Let $V$ be a non empty RLS structure and let $A$ be a subset of $V$. The functor Int $A$ yields a subset of $V$ and is defined by:

(Def. 1) $x \in \operatorname{Int} A$ iff $x \in \operatorname{conv} A$ and it is not true that there exists a subset $B$ of $V$ such that $B \subset A$ and $x \in \operatorname{conv} B$.

Let $V$ be a non empty RLS structure and let $A$ be an empty subset of $V$. Observe that $\operatorname{Int} A$ is empty.

We now state a number of propositions:

(5) For every non empty RLS structure $V$ and for every subset $A$ of $V$ holds Int $A \subseteq \operatorname{conv} A$.

(6) Let $V$ be a real linear space-like non empty RLS structure and $A$ be a subset of $V$. Then $\operatorname{Int} A=A$ if and only if $A$ is trivial.

(7) If $A \subset B$, then conv $A$ misses $\operatorname{Int} B$.

(8) $\operatorname{conv} A=\bigcup\{\operatorname{Int} B: B \subseteq A\}$.

(9) $\operatorname{conv} A=\operatorname{Int} A \cup \bigcup\{\operatorname{conv} A \backslash\{v\}: v \in A\}$.

(10) If $x \in \operatorname{Int} A$, then there exists a linear combination $L$ of $A$ such that $L$ is convex and $x=\sum L$.

(11) For every linear combination $L$ of $A$ such that $L$ is convex and $\sum L \in$ Int $A$ holds the support of $L=A$.

(12) For every linear combination $L$ of $I$ such that $L$ is convex and the support of $L=I$ holds $\sum L \in \operatorname{Int} I$.

(13) If Int $A$ is non empty, then $A$ is finite.

(14) If $v \in I$ and $u \in \operatorname{Int} I$ and $p \in \operatorname{conv} I \backslash\{v\}$ and $r \cdot v+(1-r) \cdot p=u$, then $p \in \operatorname{Int}(I \backslash\{v\})$. 


\section{The Center of Mass}

Let us consider $V$. The center of mass of $V$ yielding a function from

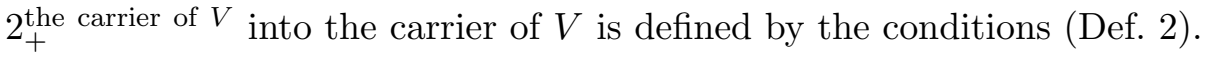

(Def. 2)(i) For every non empty finite subset $A$ of $V$ holds (the center of mass of $V)(A)=\frac{1}{\bar{A}} \cdot \sum A$, and

(ii) for every $A$ such that $A$ is infinite holds (the center of mass of $V)(A)=$ $0_{V}$.

One can prove the following propositions:

(15) There exists a linear combination $L$ of $A_{1}$ such that $\sum L=r \cdot \sum A_{1}$ and $\operatorname{sum} L=r \cdot \overline{\overline{A_{1}}}$ and $L=\mathbf{0}_{\mathrm{LC}_{V}}+\cdot\left(A_{1} \longmapsto r\right)$.

(16) If $A_{1}$ is non empty, then (the center of mass of $\left.V\right)\left(A_{1}\right) \in \operatorname{conv} A_{1}$.

(17) If $\bigcup F$ is finite, then (the center of mass of $V)^{\circ} F \subseteq \operatorname{conv} \bigcup F$.

(18) If $v \in I_{1}$, then $\left((\right.$ the center of mass of $\left.V)\left(I_{1}\right) \rightarrow I_{1}\right)(v)=\frac{1}{\overline{I_{1}}}$.

(19) (The center of mass of $V)\left(I_{1}\right) \in I_{1}$ iff $\overline{\overline{I_{1}}}=1$.

(20) If $I_{1}$ is non empty, then (the center of mass of $\left.V\right)\left(I_{1}\right) \in \operatorname{Int} I_{1}$.

(21) If $A \subseteq I_{1}$ and (the center of mass of $\left.V\right)\left(I_{1}\right) \in$ Affin $A$, then $I_{1}=A$.

(22) If $v \in A_{1}$ and $A_{1} \backslash\{v\}$ is non empty, then (the center of mass of $\left.V\right)\left(A_{1}\right)=$ $\left(1-\frac{1}{\overline{\overline{A_{1}}}}\right) \cdot(\text { the center of mass of } V)_{A_{1} \backslash\{v\}}+\frac{1}{\overline{\overline{A_{1}}}} \cdot v$.

(23) If conv $A \subseteq \operatorname{conv} I_{1}$ and $I_{1}$ is non empty and conv $A$ misses Int $I_{1}$, then there exists a subset $B$ of $V$ such that $B \subset I_{1}$ and conv $A \subseteq \operatorname{conv} B$.

(24) If $\sum L_{1} \neq \sum L_{2}$ and $\operatorname{sum} L_{1}=\operatorname{sum} L_{2}$, then there exists $v$ such that $L_{1}(v)>L_{2}(v)$.

(25) Let $p$ be a real number. Suppose $\left(r \cdot L_{1}+(1-r) \cdot L_{2}\right)(v) \leq p \leq\left(s \cdot L_{1}+\right.$ $\left.(1-s) \cdot L_{2}\right)(v)$. Then there exists a real number $r_{1}$ such that $\left(r_{1} \cdot L_{1}+(1-\right.$ $\left.\left.r_{1}\right) \cdot L_{2}\right)(v)=p$ and if $r \leq s$, then $r \leq r_{1} \leq s$ and if $s \leq r$, then $s \leq r_{1} \leq r$.

(26) If $v, u \in \operatorname{conv} A$ and $v \neq u$, then there exist $p, w, r$ such that $p \in A$ and $w \in \operatorname{conv} A \backslash\{p\}$ and $0 \leq r<1$ and $r \cdot u+(1-r) \cdot w=v$.

(27) $A \cup\{v\}$ is affinely independent iff $A$ is affinely independent but $v \in A$ or $v \notin \operatorname{Affin} A$.

(28) If $A_{1} \subseteq I$ and $v \in A_{1}$, then $(I \backslash\{v\}) \cup\{$ (the center of mass of $\left.V)\left(A_{1}\right)\right\}$ is an affinely independent subset of $V$.

(29) Let $F$ be a $\subseteq$-linear family of subsets of $V$. Suppose $\bigcup F$ is finite and affinely independent. Then (the center of mass of $V)^{\circ} F$ is an affinely independent subset of $V$.

(30) Let $F$ be a $\subseteq$-linear family of subsets of $V$. Suppose $\bigcup F$ is affinely independent and finite. Then $\operatorname{Int}\left((\text { the center of mass of } V)^{\circ} F\right) \subseteq \operatorname{Int} \bigcup F$. 


\section{REFERENCES}

[1] Grzegorz Bancerek. Cardinal numbers. Formalized Mathematics, 1(2):377-382, 1990.

[2] Grzegorz Bancerek and Krzysztof Hryniewiecki. Segments of natural numbers and finite sequences. Formalized Mathematics, 1(1):107-114, 1990.

[3] Czesław Byliński. Functions and their basic properties. Formalized Mathematics, 1(1):55$65,1990$.

[4] Czesław Byliński. Functions from a set to a set. Formalized Mathematics, 1(1):153-164, 1990.

[5] Agata Darmochwał. Finite sets. Formalized Mathematics, 1(1):165-167, 1990.

[6] Noboru Endou, Takashi Mitsuishi, and Yasunari Shidama. Convex sets and convex combinations. Formalized Mathematics, 11(1):53-58, 2003.

[7] Krzysztof Hryniewiecki. Basic properties of real numbers. Formalized Mathematics, 1(1):35-40, 1990.

[8] Jarosław Kotowicz. Real sequences and basic operations on them. Formalized Mathematics, 1(2):269-272, 1990.

[9] Beata Padlewska. Families of sets. Formalized Mathematics, 1(1):147-152, 1990.

[10] Karol Pąk. Affine independence in vector spaces. Formalized Mathematics, 18(1):87-93, 2010, doi: 10.2478/v10037-010-0012-z.

[11] Andrzej Trybulec. Domains and their Cartesian products. Formalized Mathematics, 1(1):115-122, 1990.

[12] Wojciech A. Trybulec. Linear combinations in real linear space. Formalized Mathematics, $1(3): 581-588,1990$

[13] Wojciech A. Trybulec. Partially ordered sets. Formalized Mathematics, 1(2):313-319, 1990.

[14] Wojciech A. Trybulec. Vectors in real linear space. Formalized Mathematics, 1(2):291-296, 1990.

[15] Zinaida Trybulec. Properties of subsets. Formalized Mathematics, 1(1):67-71, 1990.

[16] Edmund Woronowicz. Relations defined on sets. Formalized Mathematics, 1(1):181-186, 1990. 